\title{
Changes of Lipid Profile and Other Biological Parameters in People Living with Human Immunodeficiency Virus on Highly Active Antiretroviral Therapy in the General Referral Provincial Hospital of Bukavu, Eastern of the Democratic Republic of Congo
}

Antoine Sadiki Kishabongo ${ }^{1,2}$, Constantin Useni Shabani ${ }^{1}$, Célestin Kyambikwa Bisangamo ${ }^{2}$, Tony Akilimali Shindano ${ }^{3}$ and Ntondo B Takaisi-Kikuni ${ }^{4, *}$

${ }^{1}$ Department of Laboratory Medicine, General Referral Provincial Hospital of Bukavu, Faculty of Medicine, Catholic University of Bukavu, Bukavu, Democratic Republic of Congo

${ }^{2}$ Department of Laboratory Techniques, High Institute of Medical Techniques of Bukavu (ISTM-Bukavu), Bukavu, Democratic Republic of Congo

${ }^{3}$ Department of Internal Medicine, General Referral Provincial Hospital of Bukavu, Faculty of Medicine, Catholic University of Bukavu, Bukavu, Democratic Republic of Congo

${ }^{4}$ Laboratory of General and Pharmaceutical Microbiology, Department of Biopharmaceutical Sciences, Faculty of Pharmaceutical Sciences, University of Kinshasa, Kinshasa, Democratic Republic of Congo

*Corresponding authors: Takaisi-Kikuni NB, Laboratory of General and Pharmaceutical Microbiology, Department of Biopharmaceutical Sciences, Faculty of Pharmaceutical Sciences, University of Kinshasa, Kinshasa, Democratic Republic of Congo, Tel: +243815038118; E-mail: takaisik@yahoo.de

Received: 06 Aug, 2020 | Accepted: 24 Sep, 2020 | Published: 29 Sep, 2020

Citation: Kishabongo AS, Shabani CU, Bisangamo CK, Shindano TA, Takaisi-Kikuni NB (2020) Changes of Lipid Profile and Other Biological Parameters in People Living with Human Immunodeficiency Virus on Highly Active Antiretroviral Therapy in the General Referral Provincial Hospital of Bukavu, Eastern of the Democratic Republic of Congo. J HIV AIDS 6(2): dx.doi.org/10.16966/2380-5536.177

Copyright: (C) 2020 Kishabongo AS, et al. This is an open-access article distributed under the terms of the Creative Commons Attribution License, which permits unrestricted use, distribution, and reproduction in any medium, provided the original author and source are credited.

\section{Abstract}

Objective: Metabolic disturbances such as dyslipidemia and liver enzymes changes may be associated with the risk for developing cardiac disorder as well as liver toxicity in PLWH having HAART. This study investigated the biological pattern of PLWH on effective antiretroviral therapy in the Democratic Republic of Congo.

Methods: This was a case-control study including 45 PLWH who were going to initiate HAART and 40 drug-naive HIV-infected patients as a control group. Subjects were followed-up from baseline to six months by assessing their CD4+T cell counts and biochemical concentrations of serum cholesterols (CHOL, LDL-C, and HDL-C), TGs, aminotransferases (AST and ALT), creatinine, urea, and FPG. Atherogenic Indices (AI) were also calculated. Differences between groups were estimated using the student's $t$ test or analysis of variance if more than two groups. The $p$ values $<0.05$ was considered as statistically significant.

Results: After six months, the mean CD4+ T cell counts were significantly improved ( $p<0.0001)$ in PLWH on HAART. However, there were significant increases of CHOL, LDL-C and TG levels $(p<0.0001)$, whereas HDL-C levels were significantly decreased $(p<0.0001)$ over six months of follow-up. Higher Als were also observed in PLWH having HAART than in HAART-naive subjects $(p<0.0001)$. AST and ALT levels were higher in people living with HIV on HAART than in control group at baseline $(p=0.007$ and $p=0.002)$, three months $(p=0.059$ and $p=0.001)$ and six months ( $p=0.0140$ and $p=0.0142$ ). However, the AST and ALT differences were found more pronounced in women PLWH on HAART ( $<<0.05)$. In HIV subjects on HAART, the values of $\mathrm{s} C r$ and urea have not significantly changed over six months of follow-up ( $p=0.105$ and $p=0.06)$. There was a significant decrease of $F P G$ in people living with HIV on HAART over six months $(p<0.0001)$.

Conclusion: This study showed biochemical abnormalities in PLWH on effective antiretroviral therapy. Thus, the monitoring of biological profile would be of great importance in the clinical follow-up of HIV subjects having HARRT.

Keywords: People living with HIV; Highly active antiretroviral therapy; Biochemical profile 


\section{Introduction}

In Democratic Republic of the Congo (DR Congo), the prevalence of Human Immunodeficiency Virus (HIV) in adults (15-49 years) has decreased from $4 \%$ in 2005 to $0.8 \%$ in 2018 [1-3]. One study has estimated the HIV prevalence at about $1.2 \%$ in Bukavu, a city in the Eastern of the DR Congo [4]. The advent of effective antiretroviral therapy, also known as Highly Active Antiretroviral Therapy (HAART), has substantially reduced HIV-associated mortality and morbidity in infected patients. HAART is recommended as a standard medication for the management of HIV infection [5,6]. As the number of people living with HIV (PLWH) on antiretroviral therapy is increasing, there is a need to improve a clinical, therapeutic drug and biological monitoring in low- and middle-income countries. Some PLWH would not adhere to HARRT due to the drug-related side effects such as lipodystrophy, peripheral neuropathy and anemia. These side effects and toxicity may lead to the drug therapy change and discontinuation $[7,8]$.

Laboratory monitoring of HIV infection is essential in deciding to initiate antiretroviral therapy, assess the antiretroviral efficacy and detect drug toxicity [7]. In the past, the eligibility for antiretroviral treatment was assessed according to the World Health Organization (WHO) 2010 guidelines which recommended the initiation of HAART in patients with $\mathrm{CD} 4+\mathrm{T}$ cell counts less than 350 cells per microliter [9]. According to the recent WHO guidelines, all people diagnosed with HIV should start HAART regardless of CD4+ T cell counts and viral load measurements [10].

Despite the benefits provided by the use of HAART, it was reported biological abnormalities and the development of drug resistance $[11,12]$. In fact, biological abnormalities have already been reported in PLWH. However, these could be accentuated in people receiving HAART [13]. Hematological abnormalities are more dominated by anemia, neutropenia, and thrombocytopenia [14]. On the other hand, liver diseases are recognized as one of the causes of mortality in PLWH [15]. Liver enzymes changes and dyslipidemia are currently reported in patients receiving HAART [16]. The HIV-related dyslipidemia is a disorder characterized by the elevated plasma Triglycerides (TGs), Low Density Lipoprotein Cholesterol (LDL-C) levels and the reduced level of high density lipoprotein cholesterol (HDL-C) [13,17]. The main antiretroviral agents involved in biochemical changes include the nucleoside reverse transcriptase inhibitors, Non-Nucleoside Reverse Transcriptase Inhibitors (NNRTIs) and Protease Inhibitors (PIs) [18].

In the DR Congo, there are few studies investigating biological abnormalities observed in PLWH receiving HAART. As biological abnormalities may be associated with the risk for developing cardiac disorder and liver toxicity, this study investigated the biological pattern of people living with HIV on HAART. The control of biochemical profile will be of great importance in the clinical and biological monitoring of the PLWH.

\section{Materials and Methods}

\section{Patients}

It is a prospective case-control study carried out at the General Referral Provincial Hospital of Bukavu, from August 2012 to March 2013. This study enrolled 85 ambulatory people living with HIV aged at least 15 years: 45 PLWH who were going to initiate HAART and 40 HAART-naive PLWH in control group. The 2010 WHO eligibility criteria recommend PLWH having CD4+ T cell counts less than 350 cells $/ \mu$ L to HAART [9]. All PLWH in the study group were either at the clinical stage 2 or 3 of the HIV/AIDS disease classification [19]. The
PLWH already on antiretroviral treatment, patients with viral hepatitis and heavy alcohol consumption were excluded of this study. Data related to the presence of hypertension, body mass index, smoking, last drug used were not available in clinical settings. Before starting the investigation, the informed patient consents were obtained from all participants. This study got an approval from the ethic committee of the Catholic University of Bukavu.

\section{HAART regimens}

Different combinations of antiretroviral regimens were used at the initiation of the treatment. These consisted of (1) zidovudine (AZT) + lamivudine (3TC) + efavirenz (EFV) (20.1\%), (2) AZT+3TC+NVP (nevirapine) (64.4\%), TDF (tenofovir) $+3 \mathrm{TC}+\mathrm{EFV}(11.1 \%)$ and (3) $\mathrm{TDF}+3 \mathrm{TC}+\mathrm{NVP}(4.4 \%)$.

\section{Laboratory analyses}

All patients were checked for their serological status by a plasma qualitative rapid immunoassay test (Uni-Gold ${ }^{\text {Tx }}$ HIV, Trinity Biotech, Bray, Co Wicklow, Ireland). The confirmatory test was carried out on the Enzyme Linked Immunosorbent Assay (ELISA) analyzer (apDia $\mathrm{n} . \mathrm{v}^{\mathrm{ma}}$, Turnhout, Belgium). Biochemical analyses and CD4+ T cell counts were performed in all PLWH before the initiation of first line antiretroviral regimen, at three months and six months.

The CD4 lymphocyte $\mathrm{T}$ cell counts were estimated in plasma using the FACS Count analyzer (Becton Dickinson, San Jose, California, USA). FACS Count analyzer is a microbead-based system that is designed specifically for enumerating the absolute CD4+, CD8+ and $\mathrm{CD} 3+\mathrm{T}$-cell counts in plasma. Reagents were provided by the National Multisectorial Program HIV/AIDS Control of DR Congo.

Biochemical parameters were measured on semi-automated clinical chemistry analyzer (Cyan Smart, Cypress Diagnostics, Langdorp, Belgium). All biochemical reagents used were from Cypress Diagnostics Company. The serum Aspartate Aminotransferase (AST) and Alanine Aminotransferase (ALT) were measured using ultraviolet kinetic methods at $340 \mathrm{~nm}$. The upper limit of normal (ULN) ALT was $40 \mathrm{U} / \mathrm{L}$ in male and $32 \mathrm{U} / \mathrm{L}$ in women where as the ULN for AST activity was $38 \mathrm{U} / \mathrm{L}$ in men and $31 \mathrm{U} / \mathrm{L}$ in female $[20,21]$.

Serum analyses of total cholesterol (CHOL), HDL-C and TGs were performed on fasting using appropriate enzymatic colorimetric methods. The indirect method of Friedwald was used to estimate the LDL-C. Abnormal lipid profile was defined as CHOL $\geq 200 \mathrm{mg} / \mathrm{dL}$, HDL-C $<40 \mathrm{mg} / \mathrm{dL}, \mathrm{LDL}-\mathrm{C} \geq 130 \mathrm{mg} / \mathrm{dL}$ and TGs $\geq 150 \mathrm{mg} / \mathrm{dL}$ [22]. The Atherogenic Index (AI), calculated as the ratio of CHOL/HDL-C, was considered as a marker for predicting cardiovascular diseases [23].

Fasting plasma glucose (FPG) was measured according to the glucose oxidase method. Diabetes was defined according to the American Diabetes Association Criteria (FPG less than $126 \mathrm{mg} / \mathrm{dL}$ ) [24]. The picric acid kinetic method of Jaffe was used to estimate serum creatinine $(\mathrm{sCr})$ (adult reference range $0.9-1.3 \mathrm{mg} / \mathrm{dL}$ for men, $0.6-1.1 \mathrm{mg} / \mathrm{dL}$ for women) [25]. Urea was measured by an enzymatic method of urease (adult reference range $5-45 \mathrm{mg} / \mathrm{dL}$ ) [26].

\section{Statistical analysis}

The MedCalc software version 19.3.1 (MedCalc Software Ltd, Ostend, Belgium; https://www.medcalc.org; 2020) was used for statistical analyses. Frequencies and percentages were calculated for the categorical variables. The normality of the data was evaluated by Kolmogorov-Smirnov test. Results were expressed as mean plus or minus standard deviation (SD). Differences between groups were estimated using the Student's t-test. Chi-square test was used to 
evaluate differences in frequency distribution. The repeated measures analysis of variance allowed the comparison between more than two groups. Further, the Bonferroni post hoc analysis provided a pairwise comparison of the means. P values less than 0.05 were considered statistically significant.

\section{Results}

This study investigated the biochemical profile of 45 PLWH on HAART, with 40 HAART-naive PLWH as a control group. The mean plus or minus age (mean $\pm \mathrm{SD}$ ) between two groups was respectively $35 \pm 13$ and $34 \pm 10$ years. The majority of PLWH were women $(61.2 \%)$. The mean weight $(\mathrm{kg})$ between HAART-PLWH and control group were significantly different at the enrollment $(47.43 \pm 14.35 \mathrm{vs}$. $60.1 \pm 7.5, \mathrm{p}=0.0001)$.

\section{CD4+ T cell counts}

As shown in table 1 , the mean CD4+ T cell counts at the enrollment were significantly different between PLWH eligible to HAART and control group $(156.4 \pm 13.7$ vs. $686.5 \pm 118.5$ cell $/ \mu \mathrm{L}, \mathrm{p}<0.0001)$. However, the mean CD4+ T cell counts were significantly improved in the HAART HIV-infected cohort from baseline to six months after the initiation of antiretroviral treatment $(\mathrm{p}<0.0001)$. In contrast, these significantly decreased in HIV- infected control group.

\section{Lipid profile}

Significant differences between PLWH on HAART and those in the HAART-naive control group for CHOL, LDL-C, and TGs started only at six months after the initiation of antiretroviral therapy $(\mathrm{p}<0.0001)$, in exception for HDL-C $((p=0.710)$; Table 2$))$. These biochemical differences were also observed in both men and women groups (Table 3 ). In the cohort of HAART people living with HIV, the CHOL, LDL-C and TGs levels increased more significantly during the 6 months of follow-up $(\mathrm{p}<0.0001)$, whereas HDL-C significantly decreased $(p<0.0001)$; table 4 and figure 1 . Since these values are out of their reference limits, they would reflect abnormalities in the lipid profiles occurring in antiretroviral-treated group. In the pair wise comparison table 5, we found significant mean differences between measurements for lipids in both PLWH on HAART and naive HIV control group. In order to evaluate the cardiovascular risk of these abnormal lipid patterns, the AI were calculated. We observed in this study that the increase of the AI values during the treatment were very high in PLWH on HAART, particularly after six months ( $<<0.0001$; Table 4$)$.

\section{Liver enzymes}

The concentrations of AST and ALT were significantly higher in the PLWH on HAART than in the drug-naive control group at baseline ( $\mathrm{p}=0.007$ and $\mathrm{p}=0.002)$, three months $(\mathrm{p}=0.059$ and $\mathrm{p}=0.001)$ and after six months $((\mathrm{p}=0.0140$ and $\mathrm{p}=0.0142$; Table 2$)$. However, AST and ALT differences between PLWH on HAART and HIV-control group were found more pronounced in females $(\mathrm{p}<0.05)$. There were no significant aminotransferase changes between men PLWH on HAART and control group (Table 3 ). As shown in table 4 , slightly no significant increases of AST and ALT levels were found in PLWH on HAART after six months of follow-up. Further, the pairwise comparisons

Table 1: Characteristics of PLWH on HAART vs. HIV-control group at the enrollment and different HAART regimens.

\begin{tabular}{|c|c|c|c|c|}
\hline \multirow{2}{*}{ Variable } & Total & PLWH on HAART & HIV-Control group & \multirow{2}{*}{$p$-value } \\
\hline & $n=85(100 \%)$ & $n=45(52,9 \%)$ & $n=40(47,1 \%)$ & \\
\hline \multicolumn{5}{|l|}{ Age (years) } \\
\hline Mean (SD) & $34.2(11.8)$ & $34.6(13.4)$ & $33.9(10.2)$ & 0.780 \\
\hline$<18$ & $8(9.4)$ & $7(15.6)$ & $1(2.5)$ & \multirow{4}{*}{0.042} \\
\hline $18-30$ & $21(24.7)$ & $7(15.6)$ & $14(35)$ & \\
\hline $31-43$ & $34(40.0)$ & $17(37.8)$ & $17(42.5)$ & \\
\hline$>44$ & $22(25.9)$ & $14(31)$ & $8(20)$ & \\
\hline \multicolumn{5}{|l|}{ Sex } \\
\hline Men & $33(38.8)$ & $15(33.3)$ & $18(45)$ & \multirow{2}{*}{0.271} \\
\hline Women & $52(61.2)$ & $30(66.7)$ & $22(55)$ & \\
\hline \multicolumn{5}{|l|}{ Weight (Kg) } \\
\hline Mean (SD) & $53.6(13.3)$ & $47.43(14.35)$ & $60.1(7.5)$ & 0.0001 \\
\hline \multicolumn{5}{|c|}{ CD4+ Tcell count (cells/ $\mu l)$} \\
\hline Mean (SD) & $412.7(294.3)$ & $156.4(13.7)$ & $686.5(118.5)$ & $<0.0001$ \\
\hline$<200$ & $29(34.1)$ & $29(100)$ & $0(0.0)$ & \multirow{3}{*}{$<0.0001$} \\
\hline $200-499$ & $16(18.8)$ & $15(93.8)$ & $1(6.3)$ & \\
\hline$\geq 500$ & $40(47.1)$ & $1(2.5)$ & 39 (97.5) & \\
\hline \multicolumn{5}{|l|}{ HAART regimens } \\
\hline $\mathrm{AZT}+3 \mathrm{TC}+\mathrm{EFV}(\%)$ & - & $9(20.1)$ & - & - \\
\hline$A Z T+3 T C+N V P(\%)$ & - & $29(64.4)$ & - & - \\
\hline TDF+3TC+EFV (\%) & - & $5(11.1)$ & - & - \\
\hline TDF+3TC+NVP (\%) & - & $2(4.4)$ & - & - \\
\hline
\end{tabular}


Table 2: Mean values of weight, CD4+ T cell counts, serum cholesterols (CHOL, HDL-C, LDL-C), TGs, aminotransferases (AST and ALT), FPG, urea and $\mathrm{s} C r$ between PLWH on HAART and HIV control group at enrollment, three months and six months.

\begin{tabular}{|c|c|c|c|}
\hline & $\begin{array}{l}\text { PLWH on HAART } \\
(n=45)\end{array}$ & $\begin{array}{l}\text { HIV-Control } \\
\text { group }(n=40)\end{array}$ & \multirow{2}{*}{$\mathbf{p}$} \\
\hline & \multicolumn{2}{|c|}{ Mean (SD) } & \\
\hline \multicolumn{2}{|l|}{ At Enrollment } & & \\
\hline Weight (Kg) & $47.9(14.7)$ & $60.1(7.5)$ & $<0.0001$ \\
\hline CD4+ (cells/ $\mu \mathrm{L}$ ) & $156.4(132.7)$ & $686.5(118.5)$ & $<0.0001$ \\
\hline CHOL (mg/dL) & $144.0(46.0)$ & 165.7 (30.9) & 0.012 \\
\hline TGs (mg/dL) & 105.15 (51.3) & $102.8(24.3)$ & 0.790 \\
\hline HDL-C (mg/dL) & $41.0(14.8)$ & $44.1(32.4)$ & 0.570 \\
\hline LDL-C (mg/dL) & $82.1(39.4)$ & $100.1(31.2)$ & 0.031 \\
\hline Al (CHOL/HDL-C) & $4.2(3.9)$ & $5(3.3)$ & 0.35 \\
\hline FPG (mg/dL) & $73.7(13.2)$ & $71.2(8.5)$ & 0.290 \\
\hline AST (U/L) & $39.33(26.0)$ & $24.3(19.7)$ & 0.0007 \\
\hline ALT (U/L) & $28.63(20.0)$ & 15.5 (12.9) & 0.0002 \\
\hline UREA (mg/dL) & $20.4(8.7)$ & $21.9(7.5)$ & 0.470 \\
\hline $\mathrm{sCr}$ (mg/dL) & $1.12(0.34)$ & $1.10(0.24)$ & 0.720 \\
\hline \multicolumn{4}{|l|}{$\begin{array}{l}\text { After three } \\
\text { months of study }\end{array}$} \\
\hline Weight (Kg) & $48.3(12.7)$ & $56.8(7.4)$ & 0.0005 \\
\hline CD4+ (cells/ $\mu \mathrm{L}$ ) & $204.1(138.5)$ & $573.4(83.3)$ & $<0.0001$ \\
\hline CHOL (mg/dL) & $172.8(53.3)$ & $170.1(27.8)$ & 0.731 \\
\hline TG (mg/dL) & $114.2(42.4)$ & $109.9(27.3)$ & 0.388 \\
\hline $\mathrm{HDL}$ (mg/dL) & $29.8(13.6)$ & $33.0(20.0)$ & 0.400 \\
\hline LDL (mg/dL) & $120.1(48.1)$ & $110.9(23.2)$ & 0.220 \\
\hline Al (CHOL/HDL-C) & $7.0(4.5)$ & $6.4(3.0)$ & 0.45 \\
\hline FPG (mg/dL) & $66.9(8.8)$ & $69.0(7.5)$ & 0.290 \\
\hline AST (U/L) & 35.8 (23.49) & $26.2(19.8)$ & 0.059 \\
\hline ALT (U/L) & $26.4(17.2)$ & $18.2(12.8)$ & 0.001 \\
\hline UREA (mg/dL) & $17.8(5.8)$ & $21.2(6.5)$ & 0.033 \\
\hline $\mathrm{SCr}(\mathrm{mg} / \mathrm{dL})$ & $1.13(0.32)$ & $1.28(0.30)$ & 0.046 \\
\hline \multicolumn{4}{|l|}{$\begin{array}{l}\text { After six months } \\
\text { of study }\end{array}$} \\
\hline Weight (Kg) & $51.3(11.4)$ & $52.2(7.8)$ & 0.670 \\
\hline CD4+ (cells/ $\mu \mathrm{L}$ ) & $372.4(208.1)$ & $431.3(98.2)$ & 0.106 \\
\hline $\mathrm{CHOL}(\mathrm{mg} / \mathrm{dL})$ & $230.4(50.7)$ & $185.8(25.5)$ & $<0.0001$ \\
\hline TG (mg/dL) & $158.4(43.6)$ & $121.8(28.4)$ & $<0.0001$ \\
\hline HDL-C (mg/dL) & $27.6(12.4)$ & $26.2(20.2)$ & 0.710 \\
\hline LDL-C (mg/dL) & $173.6(44.39)$ & $130.7(21.6)$ & $<0.0001$ \\
\hline Al (CHOL/HDL-C) & $11,01(9.8)$ & $9.8(5.1)$ & 0.50 \\
\hline FPG (mg/dL) & $64.0(6.9)$ & $65.5(5.5)$ & 0.310 \\
\hline AST (U/L) & $42.08(29.6)$ & $30.3(19.4)$ & 0.0140 \\
\hline ALT (U/L) & $30.2(16.3)$ & $21.4(14.5)$ & 0.0142 \\
\hline UREA (mg/dL) & $19.5(10.7)$ & $22.9(7.1)$ & 0.150 \\
\hline $\mathrm{SCr}(\mathrm{mg} / \mathrm{dL})$ & $1.23(0.34)$ & $1.35(0.29)$ & 0.120 \\
\hline
\end{tabular}

showed no significant mean differences for AST and ALT in PLWH on HAART (Table 5). Significant increases of AST and ALT activities, but not greater than the upper normal limit, were only observed in control group (Table 4; $\mathrm{p}<0.0001$ ).

\section{Non-protein nitrogenous compounds}

Significant differences for urea and creatinine levels between PLWH on HAART and control group were observed at three months $(\mathrm{p}=0.033$ and $\mathrm{p}=0.046$ ) but not at six months (Table 2). As illustrated in table 4, no significant change was observed in $\mathrm{sCr}$ and urea in PLWH over six months of follow-up. However, $\mathrm{sCr}$ reached significantly in control group $(\mathrm{p}=0.0002)$.

\section{Fasting plasma glucose}

FPG values were not significantly different between PLWH on HAART and control group over six months (Table 2). The table 4 shows a significant decrease of serum FPG in PLWH on HAART as well as in control group during the six months of follow-up $(\mathrm{p}<0.001)$.

\section{Discussion and Conclusion}

The HIV disease is associated with a depletion of lymphocyte CD4+ T cell counts [27]. This study showed a significant increase of CD4+ T cell counts in PLWH over six months of HAART, whereas a decline in CD4+ $\mathrm{T}$ cell counts was observed in the HAARTnaive patients. Therefore, antiretroviral drugs would improve the patients' quality of life by reducing the occurrence of new acquired immunodeficiency syndrome-related opportunistic infections and deaths [27]. However, drug-related side effects are very common in PLWH. They would lead to metabolism disturbances which are generally characterized by biochemical abnormalities such as lipid and aminotransferase changes [27].

In both men and women PLWH enrolled in this study, significant differences for CHOL, LDL-C and TGs were observed between antiretroviral naive people living with HIV and those on HAART only at six months. Many studies evaluating lipid profile in PLWH receiving HAART are consistent with this finding [28-31]. HIV infection itself may have an effect on the metabolism of lipids, because we observed significant increases of total cholesterol, LDL-C and TGs values as well as a decrease of HDL-C in the drug-naive HIV-control group after six months of follow-up. The alterations in cholesterol metabolism that occur in PLWH naïve-HAART could be explained by lipid peroxidation. Modified lipid species, including oxidized forms of LDL-C and HDL-C, may contribute directly to monocyte and endothelial cell activation in HIV disease, placing them on the mechanistic pathway for increased inflammation and immune activation. These events are mediated by cytokines, including tumor necrosis factor, interleukin 1, interleukin 6 , and the interferons. The cytokine tumor necrosis factor-alpha plays a role in plasma lipoprotein peroxidation in PLWH by stimulating the production of reactive oxygen species [32,33]. An association between plasma levels of TG and circulating interferon gamma has been observed in PLWH. Interferon gamma is believed to increase TG levels by decreasing the TG clearance as well as increased hepatic de novo lipogenesis and very low-density lipoprotein cholesterol [34]. The changes of lipids were more pronounced in the cohort of PLWH receiving HAART (Figure 1). This finding confirmed evidence that patients using antiretroviral drugs for a prolonged period are four-fold more likely to develop lipid abnormalities or dyslipidemias [35,36]. Furthermore, high increase of the values of AI after six months of therapy observed in this study suggested that PLWH on HAART are at risk of cardiovascular diseases [23]. Other factors of cardiovascular diseases but not explored in this study may be type 2 
Table 3: Sex-related mean values of weight, CD4+ T cell counts, serum cholesterols, TGs, aminotransferases, FPG, urea and sCr between PLWH on HAART and HIV control group at enrollment, three and six months.

\begin{tabular}{|c|c|c|c|c|c|c|}
\hline \multirow{3}{*}{ Parameters } & \multicolumn{3}{|c|}{ Men $(n=33)$} & \multicolumn{3}{|c|}{ Women $(n=52)$} \\
\hline & $\begin{array}{l}\text { PLWH on HAART } \\
(n=15)\end{array}$ & $\begin{array}{l}\text { HIV-Control group } \\
\qquad(n=18)\end{array}$ & $p$-value & $\begin{array}{l}\text { PLWH on HAART } \\
(n=30)\end{array}$ & $\begin{array}{l}\text { HIV-Control group } \\
\qquad(n=22)\end{array}$ & $p$-value \\
\hline & \multicolumn{3}{|c|}{ Mean (SD) } & \multicolumn{3}{|c|}{ Mean (SD) } \\
\hline \multicolumn{7}{|c|}{ Enrollment } \\
\hline Weight (Kg) & $47.5(20.0)$ & $61.1(9.8)$ & 0.0165 & $48.1(11.7)$ & $59.3(5.0)$ & 0.0001 \\
\hline CD4+ (cells/ $\mu \mathrm{L})$ & $137.0(113.6)$ & $730.9(136.6)$ & $<0.0001$ & $166.1(142.2)$ & $650.2(88.9)$ & $<0.0001$ \\
\hline CHOL (mg/dL) & $145.9(36.7)$ & $164.6(38.2)$ & 0.165 & $143.0(47.9)$ & $166.6(24.1)$ & 0.039 \\
\hline TGs (mg/dL) & $103.0(39.1)$ & $101.6(33.8)$ & 0.913 & $106.8(57.4)$ & 103.7 (12.9) & 0.809 \\
\hline HDL-C (mg/dL) & $43.1(16.7)$ & $42.0(29.1)$ & 0.184 & $39.9(14.2)$ & 45.7 (34.3) & 0.419 \\
\hline LDL-C (mg/dL) & $86.3(26.1)$ & $102.3(37.7)$ & 0.176 & $81.9(43.0)$ & $98.3(25.4)$ & 0.118 \\
\hline FPG (mg/dL) & $69.5(15.7)$ & $72.8(9.3)$ & 0.457 & 75.2 (11.3) & $69.8(7.8)$ & 0.064 \\
\hline AST (U/L) & 35.4 (19.9) & $26.9(28.5)$ & 0.341 & 39.1 (27.7) & $22.0(6.9)$ & 0.007 \\
\hline ALT (U/L) & $32.5(28.2)$ & $16.1(18.2)$ & 0.051 & 24.5 (12.9) & $15.0(6.4)$ & 0.009 \\
\hline UREA (mg/dL) & $21.2(8.1)$ & $18.5(4.0)$ & 0.336 & $19.7(8.7)$ & $24.7(8.5)$ & 0.046 \\
\hline $\mathrm{sCr}(\mathrm{mg} / \mathrm{dL})$ & $0.98(0.33)$ & $1.16(0.24)$ & 0.088 & $1.18(0.33)$ & $1.05(0.24)$ & 0.112 \\
\hline \multicolumn{7}{|c|}{ Three months } \\
\hline Weight (Kg) & $54.4(10.3)$ & $56.9(9.2)$ & 0.4584 & $45.3(13.0)$ & $56.6(5.7)$ & 0.0004 \\
\hline CD4+ (cells/ $\mu \mathrm{L})$ & $195.0(115.1)$ & $592.2(93.0)$ & $<0.0001$ & $208.6(150.5)$ & $557.9(72.9)$ & $<0.0001$ \\
\hline CHOL (mg/dL) & $167.5(57.7)$ & $167.7(34.3)$ & 0.625 & $176.8(48.3)$ & $172.0(21.6)$ & 0.718 \\
\hline TGs (mg/dL) & $125.8(43.2)$ & $110.3(32.8)$ & 0.250 & $112.1(42.0)$ & $109.5(22.7)$ & 0.725 \\
\hline HDL-C (mg/dL) & $27.5(14.7)$ & $32.0(19.4)$ & 0.463 & $30.4(12.1)$ & $33.8(21.0)$ & 0.472 \\
\hline LDL-C (mg/dL) & $114.9(44.3)$ & $109.8(32.0)$ & 0.705 & $123.8(47.4)$ & $111.7(13.0)$ & 0.250 \\
\hline FPG (mg/dL) & $70.9(11.0)$ & $70.6(8.2)$ & 0.927 & $64.4(5.9)$ & $67.6(6.8)$ & 0.074 \\
\hline AST (U/L) & $26.5(17.4)$ & $29.1(28.8)$ & 0.763 & $38.4(24.1)$ & $23.9(6.8)$ & 0.009 \\
\hline ALT (U/L) & $19.5(10.6)$ & $19.8(17.9)$ & 0.963 & $28.0(18.3)$ & $16.8(6.4)$ & 0.009 \\
\hline UREA (mg/dL) & $16.3(5.1)$ & $20.0(5.5)$ & 0.053 & $18.2(6.0)$ & $22.2(7.1)$ & 0.032 \\
\hline$s C r(m g / d L)$ & $1.11(0.20)$ & $1.36(0.30)$ & 0.009 & $1.14(0.35)$ & $1.22(0.29)$ & 0.419 \\
\hline \multicolumn{7}{|c|}{ Six months } \\
\hline Weight (Kg) & $54.7(8.0)$ & $53.5(8.9)$ & 0.6814 & 49.6 (12.5) & $51.2(6.7)$ & 0.5938 \\
\hline CD4+ (cells/ $\mu \mathrm{L})$ & $383.9(207.4)$ & $448.0(90.8)$ & 0.704 & 366.7 (211.8) & 417.6 (103.9) & 0.182 \\
\hline CHOL (mg/dL) & $245.4(51.6)$ & $182.6(33.2)$ & 0.0002 & $226.9(50.8)$ & $188.3(17.4)$ & $<0.0001$ \\
\hline TGs (mg/dL) & $164.5(59.0)$ & $122.6(30.5)$ & 0.001 & $154.0(35.7)$ & $121.1(27.2)$ & 0.0007 \\
\hline HDL-C (mg/dL) & $26.7(10.4)$ & $25.8(20.1)$ & 0.870 & $27.7(13.8)$ & $26.6(20.7)$ & 0.810 \\
\hline LDL-C (mg/dL) & $185.6(50.3)$ & $128.6(28.5)$ & 0.0003 & $171.8(43.8)$ & $132.5(14.0)$ & 0.0002 \\
\hline FPG (mg/dL) & $62.2(3.1)$ & $65.8(6.1)$ & 0.049 & $64.8(7.8)$ & $65.0(4.9)$ & 0.910 \\
\hline AST (U/L) & $36.5(22.7)$ & $31.9(27.7)$ & 0.611 & $42.5(31.0)$ & $28.9(8.5)$ & 0.052 \\
\hline ALT (U/L) & $34.3(18.1)$ & $22.4(20.3)$ & 0.090 & $28.8(15.7)$ & $20.6(7.4)$ & 0.028 \\
\hline UREA (mg/dL) & $16.7(8.2)$ & $19.7(6.7)$ & 0.247 & $21.0(10.8)$ & $25.5(6.5)$ & 0.086 \\
\hline $\mathrm{sCr}(\mathrm{mg} / \mathrm{dL})$ & $1.25(0.30)$ & $1.34(0.30)$ & 0.380 & $1.25(0.35)$ & $1.36(0.29)$ & 0.237 \\
\hline
\end{tabular}


Table 4: Biochemical profiles of PLWH on HAART and HIV-control group over six months of follow-up.

\begin{tabular}{|c|c|c|c|c|c|c|c|c|}
\hline \multirow{2}{*}{$\begin{array}{c}\text { Parameters } \\
\text { Variables }\end{array}$} & \multicolumn{4}{|c|}{ PLWH on HAART $(n=45)$} & \multicolumn{4}{|c|}{ HIV-control group $(n=40)$} \\
\hline & 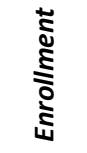 & $\sum_{m}^{\frac{n}{s}}$ & 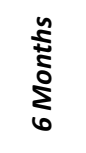 & $\begin{array}{l}\frac{1}{5} \\
\frac{1}{2} \\
2\end{array}$ & 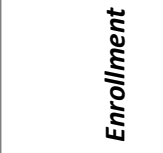 & $\frac{n}{\sum_{n}^{\frac{n}{\Sigma}}}$ & 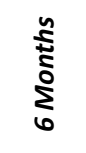 & 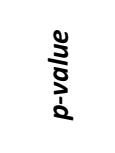 \\
\hline Weight (Kg) & 47.9 & 48.4 & 51.3 & 0.4103 & 60.1 & 56.8 & 52.2 & $<0.0001$ \\
\hline CD4+ (cells/ $\mu \mathrm{l})$ & 156.3 & 204.1 & 372.4 & $<0.0001$ & 686.5 & 573.4 & 431.3 & $<0.0001$ \\
\hline $\mathrm{CHOL}(\mathrm{mg} / \mathrm{dL})$ & 144.0 & 173.7 & 233.1 & $<0.0001$ & 165.7 & 170.1 & 185.8 & 0.0046 \\
\hline TGs (mg/dL) & 105.5 & 116.7 & 157.5 & $<0.0001$ & 102.8 & 109.9 & 121.8 & 0.0070 \\
\hline $\mathrm{HDL}-\mathrm{C}(\mathrm{mg} / \mathrm{dL})$ & 41.0 & 29.4 & 27.4 & $<0.0001$ & 44.1 & 33.0 & 26.2 & 0.0066 \\
\hline LDL-C (mg/dL) & 83.4 & 120.8 & 176.4 & $<0.0001$ & 100.1 & 110.9 & 130.7 & $<0.0001$ \\
\hline $\mathrm{Al}$ (CHOL/HDL-C) & 4.3 & 7.0 & 11.2 & $<0.0001$ & 5.0 & 6.4 & 9.8 & $<0.0001$ \\
\hline $\mathrm{FPG}(\mathrm{mg} / \mathrm{dL})$ & 73.3 & 66.6 & 63.9 & $<0.0001$ & 71.2 & 69.0 & 65.4 & 0.0021 \\
\hline AST (U/L) & 37.8 & 34.4 & 40.5 & 0.6210 & 24.3 & 26.2 & 30.3 & $<0.0001$ \\
\hline ALT (U/L) & 27.2 & 25.2 & 30.6 & 0.4000 & 15.5 & 18.2 & 30.3 & $<0.0001$ \\
\hline UREA (mg/dL) & 20.2 & 17.5 & 19.5 & 0.0607 & 21.9 & 21.2 & 22.9 & 0.5639 \\
\hline $\mathrm{sCr}(\mathrm{mg} / \mathrm{dL})$ & 1.11 & 1.13 & 1.25 & 0.1051 & 1.10 & 1.28 & 1.35 & 0.0002 \\
\hline
\end{tabular}

Table 5: Pairwise comparisons of different measurements at baseline (1), three months (2) and six months (3).

\begin{tabular}{|c|c|c|c|c|c|c|}
\hline & & & \multicolumn{2}{|c|}{ PLWH on HAART $(n=45)$} & \multicolumn{2}{|c|}{ HIV-control group $(n=40)$} \\
\hline \multicolumn{3}{|c|}{ Variables } & Mean difference & p-value & Mean difference & p-value \\
\hline \multirow{3}{*}{ Weight (Kg) } & $(1)$ & (2) & -0.43 & 1.00 & 3.67 & $<0.0001$ \\
\hline & (1) & (3) & -2.66 & 1.00 & 8.28 & $<0.0001$ \\
\hline & (2) & (3) & -3.09 & 0.530 & 4.62 & $<0.0001$ \\
\hline \multirow{3}{*}{ CD4+ (cells/ $\mu \mathrm{l})$} & (1) & (2) & -47.71 & 0.260 & 113.18 & $<0.0001$ \\
\hline & (1) & (3) & -216.04 & $<0.0001$ & 255.23 & $<0.0001$ \\
\hline & (2) & (3) & -168.33 & 0.0007 & 142.05 & $<0.0001$ \\
\hline \multirow{3}{*}{ CHOL (mg/dL) } & (1) & (2) & $-29,73$ & 0.008 & -4.38 & 0.007 \\
\hline & (1) & (3) & $-89,13$ & $<0.0001$ & -20.08 & $<0.0001$ \\
\hline & (2) & (3) & $-59,40$ & $<0.0001$ & -15.70 & $<0,0001$ \\
\hline \multirow{3}{*}{ TGs (mg/dL) } & (1) & (2) & -11.16 & 0.840 & -7.08 & 0.0017 \\
\hline & (1) & (3) & -51.96 & 0.0001 & -19.03 & $<0.0001$ \\
\hline & (2) & (3) & -40.80 & $<0.0001$ & -11.95 & $<0.0001$ \\
\hline \multirow{3}{*}{ HDL-C (mg/dL) } & (1) & (2) & 11.56 & 0.0015 & 11.08 & 0.0002 \\
\hline & (1) & (3) & 13.6 & 0.0003 & 17.83 & $<0.0001$ \\
\hline & (2) & (3) & 2.04 & 1.000 & 6.75 & $<0.0001$ \\
\hline \multirow{3}{*}{ LDL-C (mg/dL) } & (1) & (2) & -37.44 & $<0.0001$ & -10.75 & 0.0021 \\
\hline & (1) & (3) & -93.00 & $<0.0001$ & -30.60 & $<0.0001$ \\
\hline & (2) & (3) & $-55,56$ & $<0.0001$ & -19.85 & $<0.0001$ \\
\hline \multirow{3}{*}{$\mathrm{Al}$ (CHOL/HDL-C) } & (1) & (2) & -2.75 & 0.0097 & -1.39 & 0.0001 \\
\hline & (1) & (3) & -6.94 & 0.0002 & -4.84 & $<0.0001$ \\
\hline & (2) & (3) & -4.19 & 0.0313 & -3.46 & $<0.0001$ \\
\hline \multirow{3}{*}{ FPG (mg/dL) } & (1) & (2) & 6.46 & 0.012 & 2.23 & 0.065 \\
\hline & (1) & (3) & 9.00 & 0.0002 & 5.80 & 0.0003 \\
\hline & (2) & (3) & 2.55 & 0.504 & 3.58 & 0.0121 \\
\hline \multirow{3}{*}{ AST (U/L) } & (1) & (2) & 3.44 & 1.000 & -1.95 & 0.0053 \\
\hline & (1) & (3) & -2.64 & 1.000 & -6.03 & $<0.0001$ \\
\hline & (2) & (3) & -6.09 & 0.75 & -4.08 & $<0.0001$ \\
\hline \multirow{3}{*}{ ALT (U/L) } & (1) & (2) & 1.98 & 1.000 & -2.70 & $<0.0001$ \\
\hline & (1) & (3) & -3.44 & 1.000 & -5.98 & $<0.0001$ \\
\hline & (2) & (3) & -5.42 & 0.315 & -3.28 & 0.0007 \\
\hline \multirow{3}{*}{ UREA (mg/dL) } & (1) & (2) & 2.67 & 0.21 & 0.68 & 1.000 \\
\hline & (1) & (3) & 0.69 & 1.000 & -1 & 1.000 \\
\hline & (2) & (3) & -1.99 & 0.695 & -1.68 & 0.183 \\
\hline \multirow{3}{*}{$\mathrm{sCr}(\mathrm{mg} / \mathrm{dL})$} & (1) & (2) & -0.015 & 1,000 & -0.19 & 0.011 \\
\hline & (1) & (3) & -0.134 & 0,26 & -0.26 & 0.0001 \\
\hline & (2) & (3) & -0.120 & 0,29 & -0.07 & 0.671 \\
\hline
\end{tabular}



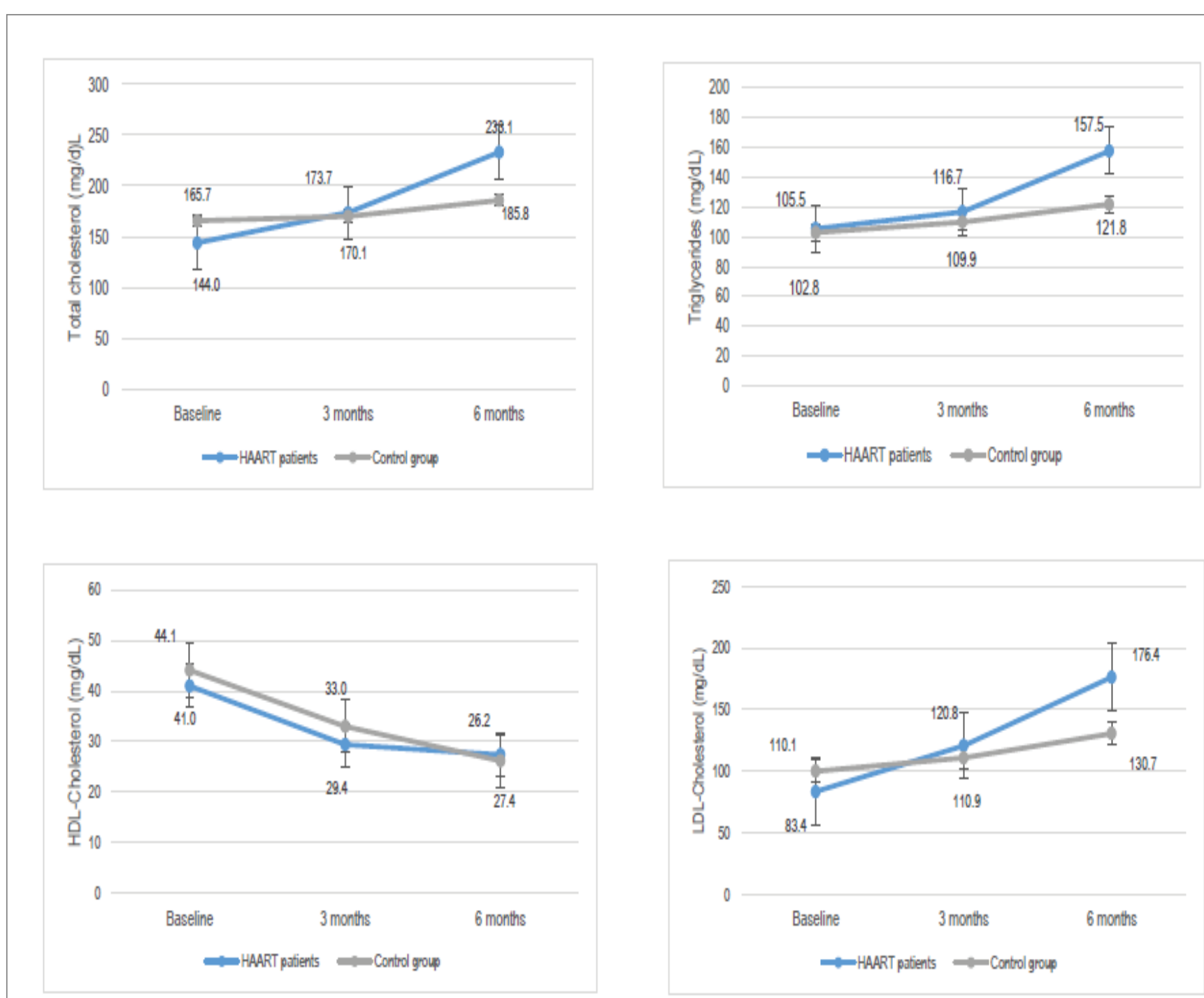

Figure 1: A more significant change of lipid profile in PLWH on HAART than in HIV control group from baseline to six months follow-up $(p<0.0001)$.

diabetes mellitus, obesity, hypertension, smoking, and family history [37]. After adjusting for these confounders, one study carried out in the Eastern DR Congo found that HAART was highly associated with metabolic syndrome, particularly with dyslipidemia in PLWH [38]. Another study carried out in the center DR Congo found that a NVPbased regimen was significantly associated with high prevalence of hypercholesterolemia in PLWH [39]. In our study, we observed that the mean values of FPG in both PLWH on HAART and control group were in reference limits (Table 2). Indeed, all patients were not on PIs, which are the most incriminated in insulin resistance occurrence [40]. Nevertheless, there was an unexplained decrease of FPG in PLWH on HAART over six months (Table 4). The patients enrolled in this study were not on alcohol abuse.

Liver toxicity is very common in HIV-infection and aminotransferases are commonly used as biomarkers of hepatocellular injury $[13,16]$. Our results showed higher ALT and AST values in PLWH on HAART than in HAART-naive control group at baseline, three months and six months (Table 2). However, AST and ALT differences between PLWH on HAART and control group were found more pronounced in women. This could be explained by the fact that women are more susceptible than men to drug-derived liver toxicity, such as alcohol and antiretrovirals [41]. Further explanation in sex difference, but not explored in this study, could be the intake of oral contraceptives [42]. It was not noticed severe aminotransferase elevations (greater than 2-5 times the ULN) in both PLWH on HAART group and drug-naive control group (Table 4). Our observations were consistent with those reported in similar studies from Uganda and Namibia which also found a lower frequency of liver enzyme elevation in PLWH on first line HAART regimens [43,21]. A longterm use (more than six months) of HAART may probably lead to important elevation of aminotransferases and can be associated with the development of chronic liver injury [16,44]. In some instances, a fulminant and early elevation of aminotransferases on HAART people living with HIV can be associated with risk factors such as chronic viral hepatitis, overdose to acetaminophen, alcohol consumption, age and tuberculosis $[45,46]$. In the selected HIV-infected cohort of this study, there was no subject with viral hepatitis. 
$\mathrm{SCr}$ and urea are non-protein nitrogenous metabolites which may also be affected by the HIV infection. In people living with HIV on HAART, the values of $\mathrm{sCr}$ and urea have not significantly changed at six months of follow-up. This was also reported in one Ghanaian study [47]. Nevertheless, a long-term use of HAART has a potential to cause renal disorders, particularly in HIV patients presenting some co-morbidities [48]. SCr and urea can also be affected by factors such as age, sex, muscles and the degree of metabolism [49]. The mean age of patients indicated that they were young. Besides, only $15 \%$ of people living with HIV on HAART were exposed to nephrotoxic HAART such as TDF [50].

Majority of PLWH received a first line regimen including AZT, 3TC and NVP. These drugs are known as potentially toxics. A mechanism underlying dyslipidemia and hepatotoxicity could be antiretroviralinduced mitochondrial alterations, which are important for the energy production and metabolism of fatty acids. The subsequent depletion of mitochondrial deoxyribonucleic acid may impair the cellular respiratory chain and eventually inhibits oxygen-dependent catabolic pathways such as pyruvate and fatty acid oxidation [51]. NVP, a current NNRTI, is frequently associated with hypersensitivity reactions, direct cholestatic injury and dyslipidemia $[39,48]$.

The findings observed in this study indicated that dyslipidemia, ALT and AST abnormalities as well as non-protein nitrogenous changes could be serious threats to PLWH on HAART, since it may lead to cardiac disorders, and to liver as well as renal damages. Thus, routine and close monitoring of lipid profile, aminotransferases and other biochemical parameters would be useful in improving a clinical follow-up of PLWH having HAART, in preventing drug toxicities and in promoting patient adherence to HAART.

\section{Limitations of the Study}

The alterations in the biochemical parameters caused by HAART would be better understood if comparisons were made with seronegative subjects. However, this study only included PLWH. Further, the study was limited to a shorter period of biochemical monitoring for PLWH.A small number of PLWH enrolled in this study may also be a limitation. Although this study found significant lipid abnormalities in PLWH on HAART, other clinical determinants not explored in this study such as body mass index, smoking, hypertension may affect the lipid profile of PLWH.

\section{Acknowledgements}

We would like to thank the dozens of volunteers who have contributed to this study and the National Multisectoral Program for HIV/AIDS Control of the DRC.

\section{References}

1. Kokolomami JHT, Kayembe PK (2018) HIV/AIDS epidemic in the Democratic Republic of the Congo: Current level of key indicators and projection by 2030. Central African Journal of Public Health 4 : 86-94.

2. World Health Organization (2020) Democratic Republic of the Congo: HIV country profile 2019.

3. World Health Organization (2005) Summary country profile for HIV/ AIDS treatment scale-up (DRCongo).

4. Riziki RB, Mwangaza SM, Kashosi TM, Misuka BM, Bayunvanye FM, et al. (2019) Screening for human immunodeficiency virus, hepatitis $B$, high blood pressure, and diabetes mellitus in the general population of South Kivu-Results of World AIDS Day 2016. World Journal of AIDS 9: 11-22.
5. Brinkhof MWG, Boulle A, Weigel R, Messou E, Mathers C, et al. (2009) Mortality of HIV-Infected patients starting antiretroviral therapy in Sub-Saharan Africa: Comparison with HIV-unrelated mortality. PLoS Med 6: e1000066.

6. Mutevedzi PC, Newell ML (2014) The changing face of the HIV epidemic in sub-Saharan Africa. Tropical Medicine \& International Health 19: 1015-1028.

7. Nuesch R, Srasuebkul P, Ananworanich J, Ruxrungtham K, Phanuphak $P$, et al. (2006) Monitoring the toxicity of antiretroviral therapy in resource limited settings: a prospective clinical trial cohort in Thailand. J Antimicrob Chemother 58: 637-644.

8. Kredo T, Van der Walt JS, Siegfried N, Cohen K (2009) Therapeutic drug monitoring of antiretrovirals for people with HIV. Cochrane Database Syst Rev 3: CD007268.

9. Konings E, Ambaw $Y$, Dilley K, Gichangi P, Arega T, et al. (2012) Implications of adopting new WHO guidelines for antiretroviral therapy initiation in Ethiopia. Bull World Health Organ 90: 659-663.

10. Ford N, Meintjes G, Vitoria M, Greene G, Chiller T (2017) The evolving role of CD4 cell counts in HIV care. Curr Opin HIV AIDS 12: 123-128.

11. Adewole OO, Eze S, Betiku Ye, Anteyi E, Wada I, et al. (2010) Lipid profile in HIV/AIDS patients in Nigeria. Afr Health Sci 10: 144-149.

12. Zdanowicz MM (2006) The Pharmacology of HIV drug resistance. Am J Pharm Educ 70: 100.

13. Calza L, Colangeli V, Manfredi R, Bon I, Carla Re M, et al. (2016) Clinical management of dyslipidaemia associated with combination antiretroviral therapy in HIV-infected patients. J Antimicrob Chemother 71: 1451-1465.

14. Masaisa F, Gahutu JB, Mukiibi J, Delanghe JR, Philippé J (2011) Anemia in human immunodeficiency virus-infected and uninfected women in Rwanda. Am J Trop Med Hyg 84: 456-460.

15. Kaspar MB, Sterling RK (2017) Mechanisms of liver disease in patients infected with HIV. BMJ Open Gastroenterol 4: e000166.

16. Ganesan M, Poluektova LY, Kharbanda KK, Osna NA (2018) Liver as a target of human immunodeficiency virus infection. World J Gastroenterol 24: 4728-4737.

17. Lo J (2011) Dyslipidemia and lipid management in HIV-infected patients. Curr Opin Endocrinol Diabetes Obes 18: 144-147.

18. Dube' MP, Stein JH, Aberg JA, Fichtenbaum CJ, Gerber JG, et al. (2003) Guidelines for the evaluation and management of dyslipidemia in human immunodeficiency virus (HIV)-infected adults receiving antiretroviral therapy: Recommendations of the HIV Medicine Association of the Infectious Disease Society of America and the Adult AIDS Clinical Trials Group. Clin Infec Dis 37: 613-627.

19. Rastogi S, Agrahari S, Singh UP, Singh A, Verma AS (2011) Clinical stages of HIV. Biolixir 1: 29-31.

20. Huang XJ, Choi YK, Im HS, Yarimaga O, Yoon E, et al. (2006) Aspartate Aminotransferase (AST/GOT) and Alanineaminotransferase (ALT/ GPT) Detection Techniques. Sensors (Basel) 6: 756-782.

21. Mataranyika PA, Kibuule D, Kalemeera F, Kaura H, Godman B, et al. (2018) Liver enzyme elevations in a cohort of HIV/AIDS patients on first-line antiretroviral therapy in Namibia: Findings and implications. Alexandria J Med 54: 49-56.

22. Hedayatnia M, Asadi Z, Zare-Feyzabadi R, Yaghooti-Khorasani M, Ghazizadeh $\mathrm{H}$, et al. (2020) Dyslipidemia and cardiovascular disease risk among the MASHAD study population. Lipids Health Dis 19: 42. 
23. Kazemi T, Hajihosseini M, Moossavi M, Hemmati M, Ziaee M (2018) Cardiovascular risk factors and atherogenic indices in an Iranian population: Birjand East of Iran. Clin Med Insights Cardiol 12: 1-6.

24. American Diabetes Association (2010) Diagnosis and classification of diabetes mellitus. Diabetes Care 33: S62-S69.

25. Vaishya R, Sarika Arora, Singh B, Mallika V (2010) Modification of Jaffe's kinetic method decreases bilirubin interference: A preliminary report. Indian J Clin Biochem 25: 64-66.

26. Salazar JH (2014) Overview of Urea and Creatinine. Lab Med 45: e19-e20.

27. Sari SP, Isnaini SR, Puspitasari AW (2018) Monitoring side effects of antiretroviral therapy in patients with immunodeficiency virus/ acquired immunodeficiency syndrome. Int J App Pharm 10: 321-324.

28. KumarA, Sathain B (2011) Assessment of lipid profile in patients with human immunodeficiency virus (HIV/AIDS) without antiretroviral therapy. Asian PJ Trop Dis 1: 24-27.

29. Fontas E, Leth FV, Sabin CA, Friis-Møller N, Rickenbach M, et al. (2004) Lipid profiles in HIV-infected patients receiving combination antiretroviral therapy: are different antiretroviral drugs associated with different lipid profiles? J Infect Dis 189: 1056-1074.

30. Abebe M, Kinde S, Belay G, Gebreegziabxier A, Challa F, et al. (2014) Antiretroviral treatment associated hyperglycemia and dyslipidemia among HIV infected patients at Burayu Health Center, Addis Ababa, Ethiopia: A cross-sectional comparative study. BMC Res Notes 7: 380 .

31. Indumati V, Vijay V, Shekhanawar MS, Rajeshwari, Amareshwaras $M$, et al. (2014) Comparison of serum lipid profile in HIV positive patients on ART with ART naive patients. J Clin Diagn Res 8: CC06cC09.

32. Oka F, Naito T, Oike M, Imai R, Saita M, et al. (2012) Correlation between HIV disease and lipid metabolism in antiretroviral-naive HIV-infected patients in Japan. J Infect Chemother 18: 17-21.

33. Funderburg NT, Mehta NN (2016) Lipid abnormalities and inflammation in HIV Infection. Curr HIV/AIDS Rep 13: 218-225.

34. Grunfeld C, Pang M, Doerrler W, Shigenaga JK, Jensen P, et al. (1992) Lipids, lipoproteins, triglyceride clearance, and cytokines in Human Immunodeficiency Virus Infection and the Acquired Immunodeficiency Syndrome. J Clin Endocrinol Metab 74: 10451052.

35. Ombeni W, Kamuhabwa AR (2016) Lipid profile in HIV-infected patients using first-line antiretroviral drugs. J Int Assoc Provid AIDS Care 15: 164-171.

36. Singh J, Verma M, Ghalaut PS, Verma R, Soni A, et al. (2014) Alteration in lipid profile in treatment-naive HIV- infected patients and changes following HAART initiation in Haryana. J Endocrinol Metab 4: 25-31.

37. Estrada V, Portilla J (2011) Dyslipidemia related to antiretroviral therapy. J AIDS Rev 13: 49-56.
38. Katoto PDMC, Thienemann F, Bulabula ANH, Esterhuizen TM, Murhula AB, et al. (2018) Prevalence and risk factors of metabolic syndrome in HIV-infected adults at three urban clinics in a postconflict setting, eastern Democratic Republic of the Congo. Trop Med Int Health 23: 795-805.

39. Mukeba-Tshialala D, Nachega JB, Mutombo-Tshingwali M, Arendt V, Gilson G, et al. (2017) Obesity, high blood pressure hypercholesterolemia, and untreated diabetes in HIV-infected and HIV-uninfected adults in Mbuji-Mayi (Democratic republic of congo). Bull Pathol Exot 110: 301-309.

40. Das S (2011) Insulin resistance and diabetes in HIV infection. Recent Pat Antiinfect Drug Discov 6: 260-268.

41. Guy J, Peters MG (2013) Liver disease in women: The Influence of gender on epidemiology, natural history and patient outcomes. Gastroenterol Hepatol 9: 633-639.

42. Nunez M (2006) Hepatotoxicity of antiretrovirals: incidence, mechanisms and management. J Hepatol 44: S132-139.

43. Ocama P, Castelnuovo B, Kamya MR, Kirk GD, Reynolds SJ, et al. (2010) Low frequency of liver enzyme elevation in HIV-infected patients attending a large urban treatment centre in Uganda. Int J STD AIDS 21: 553-557.

44. Alghamdi S, Alrbiaan A, Alaraj A, Alhuraiji A, Alghamdi M, et al. (2016) Elevated Alanine aminotransferase levels in HIV-infected persons without hepatitis B or C virus co-infection. Ann Saudi Med 36: 288-291.

45. Nunez M, Lana R, Mendoza JL, Martin-Carbonero L, Soriano V (2001) Risk factors for severe hepatic injury after introduction of highly active antiretroviral therapy. J Acquir Immune Defic Syndr 27: 426431.

46. Osakunor DNM, Obirikorang C, Fianu V, Asare I, Dakorah M (2015) Hepatic enzyme alterations in HIV patients on antiretroviral therapy: A case-control study in a hospital setting in Ghana. Plos One 10: e0134449.

47. Obirikorang C, Osakunor DN, Ntaadu B, Adarkwa OK (2014) Renal function in Ghanaian HIV-infected patients on highly active antiretroviral therapy: a case-control study. Plos One 9: e99469.

48. Wondifraw Baynes H, Tegene B, Gebremichael M, Birhane G, Kedir W, et al. (2016) Assessment of the effect of antiretroviral therapy on renal and liver functions among HIV-infected patients: a retrospective study. HIV AIDS (Auckl) 9: 1-7.

49. Finney $H$, Newman DJ, Price CP (2000) Adult reference ranges for serum cystatin $C$, Creatinine and predicted creatinine clearance. Ann Clin Biochem 37: 49-59.

50. Alfano G, Cappelli G, Fontana F, Di Lullo L, Di lorio B, et al. (2019) Kidney Disease in HIV infection. J Clin Med 8: 1254.

51. Spengler U, Lichterfeld M, Rockstroh JK (2002) Antiretroviral drug toxicity-a challenge for the hepatologist? J Hepatol 36: 283-294. 